\title{
Polynomial Time Approximation Schemes
}

Hadas Shachnai

Computer Science Department,

The Technion, Haifa, Israel.

E-mail: hadas@cs.technion.ac.il.
Tami Tamir

School of Computer Science, The Interdisciplinary Center, Herzliya, Israel. E-mail: tami@idc.ac.il.

October 6, 2005

\section{Introduction}

Let $\Pi$ be an NP-hard optimization problem, and let $A$ be an approximation algorithm for $\Pi$. For an instance $I$ of $\Pi$, let $A(I)$ denote the objective value when running $A$ on $I$, and let $O P T(I)$ denote the optimal objective value. The approximation ratio of $A$ for the instance $I$ is $R_{A}(I)=A(I) / O P T(I)$, thus, when $\Pi$ is minimization (maximization) problem $R_{A}(I) \geq 1\left(R_{A}(I) \leq 1\right)$.

A polynomial time approximation scheme is an algorithm which takes as input an additional parameter, $\varepsilon$, which determines the desired approximation ratio. This ratio can be arbitrarily close to 1 , when $\varepsilon$ approaches 0 . The time complexity of the scheme is polynomial in the input size but may be exponential in $1 / \varepsilon$. This gives a clear trade-off between running time and quality of approximation. Formally,

Definition 1.1 An approximation scheme for an optimization problem $\Pi$ is an algorithm $A$ which takes as input both the instance $I$ and an error bound $\varepsilon$, runs in time polynomial in $|I|$ and has approximation ratio $R_{A}(I, \varepsilon) \leq(1+\varepsilon)$. In fact, such an algorithm $A$ is a family of algorithms $A_{\varepsilon}$ such that for any instance $I$, $R_{A_{\varepsilon}}(I) \leq(1+\varepsilon)$.

The approximation algorithm $A$ may be deterministic or randomized. In the latter case the result is a randomized approximation scheme. 
Definition 1.2 A randomized approximation scheme for an optimization problem $\Pi$ is an algorithm $A$ which takes as input both the instance $I$ and an error bound $\varepsilon$, runs in time polynomial in $|I|$ and has an expected approximation ratio $R_{A}(I, \varepsilon) \leq(1+\varepsilon)$. In fact, such an algorithm $A$ is a family of algorithms $A_{\varepsilon}$ such that for any instance $I, \operatorname{EXP}\left[R_{A_{\varepsilon}}(I)\right] \leq(1+\varepsilon)$.

In some approximation schemes, an additive constant $k$, whose value is independent of $I$ and $\varepsilon$, is added to the approximation ratio. Asymptotically, this constant is negligible, thus, such a scheme is called an asymptotic PTAS.

Definition 1.3 An asymptotic approximation scheme for an optimization problem $\Pi$ is a family of algorithms $A_{\varepsilon}$ that run in polynomial time such that, for some constant $k$, and for any instance $I, A_{\varepsilon}(I) \leq$ $(1+\varepsilon) O P T(I)+k$.

We refer the reader to Chapter R-12 in this book for a detailed study of such schemes.

Some approximation algorithms provide a solution for a relaxed instance of the problem. For example, in packing problems, an algorithm may pack the items in bins whose sizes are slightly larger than the original. The objective value is achieved relative to the relaxed resource. This type of approximation algorithm is called a dual approximation algorithm [23] or approximation with resource augmentation [8]. A dual approximation scheme is a family of algorithms $A_{\varepsilon}$ that run in polynomial time, such that for any instance $I, A(I) \leq(1+\varepsilon) O P T(I)$, and $A(I)$ is achieved for resources augmented by factor of $(1+\varepsilon)$.

Depending on the function $f(|I|, 1 / \varepsilon)$ which gives the running time of the scheme, some schemes are classified as quasi-polynomial and others as fully polynomial. In particular, when the running time is $O\left(n^{\text {polylog(n)}}\right)$ we get a quasi PTAS (see, e.g. [4], [13]); when the running time is polynomial in both $|I|$ and $1 / \varepsilon$ we get a fully polynomial time approximation scheme (FPTAS). Such schemes are studied in detail in Chapter R-11.

There is a wide literature presenting PTASs for NP-hard problems. Many of these works present PTASs for certain problems/subclasses of problems. While some of the proposed schemes may have running times which render them inefficient in practice, these works essentially help identify the class of problems that admit PTAS. There have been also some studies towards characterizing this class of problems (see, e.g. [33], [48] and Chapter R-14 in this book). We focus here on the techniques that have been repeatedly used in developing PTASs. 
We refer the reader also to the comprehensive survey on Approximation Algorithms by Motwani [37], a tutorial by Schuurman and Woeginger [42], and the survey on scheduling by Karger, Stein and Wein [29], from which we borrowed some of the examples in this chapter.

\section{Partial Enumeration}

\subsection{Extending Partial Small-Size Solutions}

There are two main techniques that are based on extending partial small-size solutions. The first technique exploits our ability to solve the problem optimally on a constant-size subset of the instance. Thus, initially, such a constant-size subset is selected. This subset contains the most 'important' elements in the instance. We identify elements as important depending on the problem at hand. The problem is solved optimally for this subset. This can be done by exhaustive search, since there is only a constant number of elements to consider. Next, this optimal partial solution is extended into a complete solution, using some heuristic with a bounded approximation ratio.

In the second technique, none of the elements is identified as 'important' initially; instead, all partial solutions of constant-size are considered, and each is extended to a complete solution using some heuristic. The best extension is selected as the PTAS's output.

The time complexity analysis of such PTASs is based on the fact that the number of possible subsets or solutions that are considered is exponential in the (constant) size of these subsets. The step in which the constant-size partial solution is extended is usually based on some greedy rule that may require sorting, and is polynomial. The parameter $\varepsilon$ specifying the required approximation ratio of $(1+\varepsilon)$ determines the size $k$ of the partial solution for which an exponential exhaustive search is applied. This implies that the running time of such schemes is exponential in $1 / \varepsilon$.

\subsubsection{Extend an Optimal Solution for a Single Subset}

We demonstrate the first technique for the problem of finding the minimum makespan (completion time) of a schedule of $n$ jobs on $m$ identical machines. The idea in this PTAS of Graham [21] is to schedule first 
optimally the $k$ longest jobs and then schedule, using some heuristic, the remaining jobs. Formally, the input for the minimum makespan problem consists of $n$ jobs and $m$ identical machines. The goal is to schedule the jobs non-preemptively on the machines in a way that minimizes the maximum completion time of any job in the schedule.

Denote by $p_{1}, \ldots, p_{n}$ the processing times of the jobs. Assume that $n>m$, and that the processing times are sorted in non-increasing order, that is, for all $i<j, \quad p_{i} \geq p_{j}$. A well-known heuristic for the makespan problem is the LPT rule, which selects the longest unscheduled job in the sorted list and assigns it to a processor currently having the minimum load. The PTAS combines an optimal schedule of the longest $k$ jobs with the LPT rule applied to the remaining jobs.

Formally, for any $k \in[0, n]$, the algorithm $A_{k}$ is defined as follows:

1. Schedule optimally, and with no intended idles, the first $k$ jobs.

2. Add the remaining jobs greedily using the LPT rule.

Theorem 1.1 Let $A_{k}(I)$ denote the makespan achieved by $A_{k}$ on an instance $I$, and let $O P T(I)$ denote the minimum makespan of $I$, then

$$
A_{k}(I) \leq O P T(I)\left(1+\frac{1-\frac{1}{m}}{1+\lfloor k / m\rfloor}\right) .
$$

\section{Proof}

Let $T$ denote the makespan of an optimal schedule of the first $k$ jobs. Clearly, $T$ is a lower bound for $O P T(I)$, thus, if the makespan is not increased in the second step, that is, $A_{k}(I)=T$, then $A_{k}$ is optimal for $I$. Otherwise, the makespan of the schedule is larger than $T$. Let $j$ be the job to determine the makespan (the one who completes last). By definition of LPT, this implies that all the machines were busy when job $j$ started its execution (otherwise job $j$ could start earlier). Since the optimal schedule from step 1 has no intended idles, it holds that all the machines are busy during the time interval $\left[0 ; A_{k}(I)-p_{j}\right]$

Let $P=\sum_{j=1}^{n} p_{j}$ be the total processing time of the $n$ jobs. By the above, $P \geq m\left(A_{k}(I)-p_{j}\right)+p_{j}$. Also, since the jobs are sorted in nonincreasing order of processing times, we have that $p_{j} \leq p_{k+1}$ and therefore $P \geq m A_{k}(I)-(m-1) p_{k+1}$. A lower bound for the optimal solution is a schedule in which the load on the $m$ machines is balanced; thus $O P T(I) \geq P / m$, which implies that $A_{k}(I) \leq O P T(I)+\left(1-\frac{1}{m}\right) p_{k+1}$. 
In order to bound $A_{k}(I)$ in terms of $O P T(I)$, we need to bound $p_{k+1}$ in terms of $O P T(I)$. To obtain such a bound, consider the $k+1$ longest jobs. In an optimal schedule, some machine is assigned at least $\lceil(k+1) / m\rceil \geq 1+\lfloor k / m\rfloor$ of these jobs. Since each of these jobs has processing time at least $p_{k+1}$, we conclude that $O P T(I) \geq(1+\lfloor k / m\rfloor) p_{k+1}$, which implies that $p_{k+1} \leq O P T(I) /(1+\lfloor k / m\rfloor)$. It follows that

$$
A_{k}(I) \leq O P T(I)\left(1+\frac{\left(1-\frac{1}{m}\right)}{(1+\lfloor k / m\rfloor)}\right)
$$

To observe that the above class of algorithms is a PTAS, we relate the value of $k$ to $(1+\varepsilon)$, the required approximation ratio: Given $\varepsilon>0$, let $k=\left\lceil\frac{1-\varepsilon}{\varepsilon} m\right\rceil$. It is easy to verify that the corresponding $A_{k}$ achieves approximation ratio at most $(1+\varepsilon)$. Thus, we conclude that for a fixed $m$, there is a polynomial time approximation scheme for the $m$ processor scheduling problem.

Note that for any fixed $k$, an optimal schedule of the first $k$ jobs can be found in $O\left(m^{k}\right)$ steps. Applying the LPT rule takes additional $O(n \log n)$. For $A_{\varepsilon}$, we get that the running time of the scheme is $O\left(m^{m / \varepsilon}\right)$, that is, exponential in $m$ (that is assumed to be constant) and $1 / \varepsilon$. This demonstrates the basic property of approximation schemes: a clear trade-off between running time and quality of approximation.

\subsubsection{Extend All Possible Solutions for Small Subsets}

The second technique, of considering all possible subsets, is illustrated in an early PTAS of Sahni for the knapsack problem [40]. An instance of the knapsack problem consists of $n$ items, each having a specified size and a profit, and a single knapsack, having size $B$. Denote by $s_{i} \geq 0, p_{i} \geq 0$ the size and profit associated with item $i$. The goal is to find a subset of the items such that the total size of the subset does not exceed the knapsack capacity, and the total profit associated with the items is maximized.

The PTAS in [40] is based on considering all $O\left(k n^{k}\right)$ possible subsets of size at most $k$, where $k$ is some fixed constant. Each of these subsets is extended to a larger feasible subset by adding more items to the knapsack, using some greedy rule. The best extension among these $O\left(k n^{k}\right)$ candidates is selected to be the output of the scheme. Formally, for any $k \in[0, n]$, the algorithm $A_{k}$ is defined as follows:

1. (Preprocessing) Sort the items in non increasing order of their profit densities, $p_{i} / s_{i}$. 
2. For each feasible subset of at most $k$ items,

(a) pack the subset in the knapsack.

(b) Add to the knapsack items in the sorted list one by one, while there is enough available space.

3. Select the best packing achieved in step 2 .

Theorem 1.2 Let $P\left(A_{k}\right)$ denote the profit achieved by $A_{k}$, and let $P(O P T)$ denote the optimal profit, then

$$
O P T\left(A_{k}\right) \leq P(A)\left(1+\frac{1}{k}\right)
$$

\section{Proof}

Let $O P T$ be any optimal solution. If $|O P T| \leq k$ we are done, since the subset $O P T$ will be considered at some iteration. Otherwise, let $H=\left\{a_{1}, a_{2}, \ldots, a_{k}\right\}$ be the set of $k$ most profitable items in OPT. There exists an iteration of $A_{k}$ in which $H$ is considered. We show that the profit gained by $A_{k}$ in this iteration yields the statement of the theorem. Consider the list $L_{1}=O P T \backslash H=\left\{a_{k+1}, \ldots, a_{x}\right\}$ of the remaining items of $O P T$, in the order they are considered by $A_{k}$. Recall that, at some point, $A_{k}$ will try $H$ as the initial set of $k$ packed items. The algorithm will then add greedily items, as long as the capacity constraint allows. If all the items are packed, $A_{k}$ is clearly optimal; otherwise, at some point there is not enough space for the next item. Let $m$ be the index of the first item in $L_{1}$ which is not packed in the knapsack by $A_{k}$, i.e. items $a_{k+1}, \ldots, a_{m-1}$ are packed. The item $a_{m}$ is not packed because $B_{e}$, the remaining empty space at this point, is smaller than $s_{m}$. The greedy algorithm packed into the knapsack only items with profit density at least $p_{m} / s_{m}$. At this time, when $a_{m}$ is rejected, the knapsack contains the items from $H$, the items $a_{k_{1}+1}, \ldots, a_{m-1}$ and some items which are not in $O P T$.

Let $G$ denote the items packed in the knapsack so far by the greedy stage of $A_{k}$. All these items have profit density at least $p_{m} / s_{m}$. In particular, the items in $G \backslash O P T$ that have total size $\Delta=B-\left(B_{e}+\sum_{i=1}^{m-1} s_{i}\right)$ all have profit density at least $p_{m} / s_{m}$. Thus, the total profit of the items in $G$ is $P(G) \geq \sum_{i=k_{1}+1}^{m-1} p_{i}+\Delta \frac{p_{m}}{s_{m}}$. We conclude that the total profit of the items in $O P T$ is

$$
\begin{aligned}
P(O P T) & =\sum_{i=1}^{k} p_{i}+\sum_{i=k+1}^{m-1} p_{i}+\sum_{i=m}^{|O P T|} p_{i} \\
& \leq P(H)+\left(P(G)-\Delta \frac{p_{m}}{s_{m}}\right)+\left(B-\sum_{i=1}^{m-1} s_{i}\right) \frac{p_{m}}{s_{m}}
\end{aligned}
$$




$$
=P(H)+P(G)+B_{e} \frac{p_{m}}{s_{m}}<P(H \cup G)+p_{m}
$$

Since $A_{k}$ packs at least $H \cup G$, we get that $P\left(A_{k}\right) \geq P(H)+P(G)$, which implies that $P(O P T)-P\left(A_{k}\right)<$ $p_{m}$. Given that there are at least $k$ items with a profit at least as large as $a_{m}$ (those selected to $H$ ), we conclude that $s_{m} \leq S(O P T) /(k+1)$. This gives the approximation ratio.

Assuming a single preprocessing in which the items are sorted by their profit densities, each subset is extended to a maximal packing in time $O(n)$. Since there are $O\left(k n^{k}\right)$ possible subsets to consider, the total running time of the scheme is $O\left(k n^{k+1}\right)$.

To obtain a PTAS for the knapsack problem, let $A_{\varepsilon}$ be the algorithm $A_{k}$ with $k=\lceil 1 / \varepsilon\rceil$. By the above, the approximation ratio is at most $1+\varepsilon$, and the running time of $A_{\varepsilon}$ is $O\left(\frac{1}{\varepsilon} n^{1+\frac{1}{\varepsilon}}\right)$.

As shown in Section 3.2, this technique, of choosing the best among a small number of partial packings, was applied also to variants of multidimensional packing.

\subsection{Applying Enumeration to a Compacted Instance}

In this section we present the technique of applying exhaustive enumeration to a modified instance, in which we have a more compact representation of the input. Approximation schemes that are based on this approach consist of three steps:

1. The instance $I$ is modified to a simpler instance, $I^{\prime}$. The parameter $\varepsilon$ determines how rough $I^{\prime}$ is in comparison with $I$. The smaller $\varepsilon$ the more refined $I^{\prime}$ is.

2. The problem is solved optimally on $I^{\prime}$.

3. An approximate solution for $I$ is induced from the optimal solution for $I^{\prime}$.

The challenge is to modify $I$ in the first step into an instance $I^{\prime}$ that is simple enough to be solved in polynomial time, yet not too different from the original $I$, so that we can use an exact solution for $I^{\prime}$ to derive an approximate solution for $I$.

The use of this technique usually involves partitioning the input into significant and and non-significant elements. The partition depends on the problem at hand. For example, it is natural to distinguish between long and short jobs in scheduling problems, and between big and small, or high-profit and low-profit elements, 
in packing problems. For a given instance, the distinction between the two types of elements usually depends on the input parameters (including $\varepsilon$ ), and on the value of an optimal solution.

In some cases, the transformation from $I$ to $I^{\prime}$ involves only grouping the non-significant elements. Each group of such elements thus forms a single significant element in $I^{\prime}$. As a result, the instance $I^{\prime}$ consists of a small number of significant elements. More details and an example for this type of transformation is given in Section 2.2.1.

In other cases, all the elements, or only the significant ones, are transformed into a set of elements with a small number of distinct values. This approach is described and demonstrated in Section 2.2.2.

\subsubsection{Grouping Subsets of Elements}

We demonstrate this technique with Sahni's PTAS [41] for the minimum makespan problem on two identical machines. The input consists of $n$ jobs with processing times $p_{1}, \ldots, p_{n}$. The goal is to schedule the jobs on two identical parallel machines in a way that minimizes the latest completion time, that is, in a way that balances the load on the two machines.

Let $P=\sum_{j=1}^{n} p_{j}$ denote the total processing time of all jobs, and let $p_{\max }$ denote the longest processing time of a job. Let $C=\max \left(P / 2, p_{\max }\right)$, Note that $C$ is a lower bound on the minimum makespan (that is $O P T \geq C$ ), since $P / 2$ is the schedule length if the load is perfectly balanced between the two machines, and since some machine must process the longest job.

The first step of the scheme is to modify the instance $I$ into a simplified instance $I^{\prime}$. This modification depends on the value of $C$ and on the parameter $\varepsilon$, and is done as follows. Given $I, \varepsilon$, partition the jobs into small jobs - of length at most $\varepsilon C$, and big jobs - of length larger than $\varepsilon C$. Let $P_{S}$ denote the total length of small jobs. The modified instance $I^{\prime}$ consists of all big jobs in $I$ together with $\left\lfloor P_{S} /(\varepsilon C)\right\rfloor$ jobs of length $\varepsilon C$.

Next, we need to solve optimally the minimum makespan problem for the instance $I^{\prime}$. Note that all jobs in $I^{\prime}$ have length at least $\varepsilon C$ and their total size is at most $P$, the total processing time of the jobs in the original instance, since the small jobs in $I$ are replaced in $I^{\prime}$ by jobs of length $\varepsilon C$ with total length at most $P_{S}$. Therefore, the number of jobs in $I^{\prime}$ is at most the constant $P / \varepsilon C \leq 2 / \varepsilon$. An optimal schedule of a constant number of jobs can be found by exhaustive search over all $O\left(2^{2 / \varepsilon}\right)$ possible schedules. This 
constant number is independent of $n$, but grows exponentially with $\varepsilon$ - as we expect from our PTAS. Denote by $O P T\left(I^{\prime}\right)$ the length of the optimal schedule.

Finally, given an optimal schedule of $I^{\prime}$, we need to transform it into a schedule of $I$. Note that, for the makespan objective, we are only concerned about the partition of the jobs between the machines, while the order in which the jobs are scheduled on each machine does not matter. To obtain a schedule in $I$, the big jobs of $I$ are scheduled on the same machine as in the optimal schedule for $I^{\prime}$. The small jobs are scheduled greedily in an arbitrary order on the first machine until for the first time, the total load on the first machine is at least $O P T\left(I^{\prime}\right)$. The remaining small jobs are scheduled on the second machine. Clearly, the overflow on the first machine is at most $\varepsilon C$ (maximal length of a small job). Also, since the total number of $(\varepsilon C)$-jobs was determined to be $\left\lfloor P_{S} /(\varepsilon C)\right\rfloor$, the overflow on the second machine is also bounded by $\varepsilon C$. Therefore, the resulting makespan of $I^{\prime}$ 's schedule is at most $O P T\left(I^{\prime}\right)+\varepsilon C$.

To complete the analysis we need to relate $O P T\left(I^{\prime}\right)$ to $O P T(I)$.

Claim 1.3 OPT $\left(I^{\prime}\right) \leq(1+\varepsilon) O P T(I)$

\section{Proof}

Given a schedule of $I$, in particular an optimal one, a schedule for $I^{\prime}$ can be derived by replacing, on each machine separately, the small jobs with jobs of size $\varepsilon C$, with at least the same total size. Recall that the number of $(\varepsilon C)$-jobs in $I^{\prime}$ was determined to be $\left\lfloor P_{S} /(\varepsilon C)\right\rfloor$. Independent of the partition of the small jobs of $I$ between the two machines, the result of this replacement is a feasible schedule of $I^{\prime}$ whose makespan is at most $O P T(I)+\varepsilon C$. Since $O P T(I) \geq C$, the statement of the claim holds.

Back to our scheme, we showed that the optimal schedule of $I^{\prime}$ is converted into a feasible scheme of $I$ with makespan at most $O P T\left(I^{\prime}\right)+\varepsilon C$. By the above claim, this value is at most $(1+\varepsilon) O P T(I)+\varepsilon C \leq$ $(1+2 \varepsilon) O P T(I)$

By selecting $\varepsilon^{\prime}=\varepsilon / 2$, and running the scheme for $\varepsilon^{\prime}$, we get the desired ratio of $(1+\varepsilon)$.

This scheme can be extended to any constant number of machines. For an arbitrary number of machines, a more complex PTAS, that requires reducing the number of distinct values in the input [23], exists and is given in the next section. 


\subsubsection{Reducing the Number of Distinct Values in the Input}

Any optimization problem can be solved optimally in polynomial or even constant time if the input size is some constant. For many optimization problems, an efficient algorithm exists if the input size is arbitrary but the number of distinct values in the input is a constant. Alternatively, the problem can be solved by a pseudo-polynomial-time algorithm (e.g., via dynamic programming), whose running time depends on the instance parameters, and is therefore polynomial only if the parameter values are polynomial in the problem size.

The idea behind the technique described below is to transform the elements (or sometimes, only the significant elements) in the instance $I$ into an instance $I^{\prime}$ in which the number of distinct values is fixed, or to scale the values according to the input size. The problem is then solved on $I^{\prime}$, and the solution for $I^{\prime}$ is transformed to a solution for the original instance. The non-significant elements, which are sometimes omitted from $I^{\prime}$, are added later to the solution, using some heuristic. The parameter $\varepsilon$ determines the (constant) number of distinct values contained in $I^{\prime}$ : the smaller $\varepsilon$, the larger number of distinct values. There are two main approaches for determining the values in $I^{\prime}$.

1. Rounding - The values of $I^{\prime}$ form an arithmetic series in which the difference between elements is a function of $\varepsilon$. For example, multiples of $\varepsilon^{2} T$, for some value $T$. In this approach, the gap between any two values bounds the difference between an original value of an element in $I$ and the value of the corresponding element in $I^{\prime}$. Note that the amounts of elements rounded to the different values in $I^{\prime}$ is arbitrarily distributed.

2. Shifting - The values of $I^{\prime}$ are a subset of the values of $I$, selected such that the number of elements in $I$ that are represented by one value is uniform. On the other hand, unlike the rounding approach, there is no bound on the difference between the value of an element in $I$ and its corresponding element in $I^{\prime}$. For example, partition the element into $\left\lceil 1 / \varepsilon^{2}\right\rceil$ groups, each having at most $\left\lfloor n / \varepsilon^{2}\right\rfloor$ elements, and fix the values in each group to be (say) the minimal value of an element in the group.

In both approaches, the approximation ratio is guaranteed to be $(1+\varepsilon)$ if $I^{\prime}$ is close enough to $I$. Formally, an optimal solution for $I^{\prime}$ induces a solution for $I$ whose value is bigger/smaller by a factor of at most $(1+\varepsilon)$. 
Another factor of $(1+\varepsilon)$ to the approximation ratio may be added due to the non-significant items - in the case they are handled separately.

We demonstrate this technique with the classic PTAS of Hochbaum and Shmoys [23] for the minimum makespan problem on parallel machines. Recall that the input is a set of $n$ jobs having processing times $p_{1}, \ldots, p_{n}$, and $m$ identical machines; the goal is to schedule the jobs on the machines in a way that minimizes the latest completion time. The number of machines, $m$, can be arbitrarily large (otherwise, a simpler PTAS exists; see Section 2.2.1).

First, note that the minimum makespan (MM) problem is closely related to the bin packing (BP) problem. The input for $\mathrm{BP}$ is a collection of items with sizes between 0 and 1 . The goal is to pack the items using a minimal number of bins. Formally, let $I=\left\{p_{1} \ldots p_{n}\right\}$ be the sizes in a set of $n$ items where $0 \leq p_{j} \leq 1$. The goal is to find a collection of subsets $U=\left\{B_{1}, B_{2}, \ldots, B_{k}\right\}$ which forms a disjoint partition of $I$, such that for all $i, 1 \leq i \leq k, \sum_{j \in B_{i}} p_{j} \leq 1$, and the number of bins, $k$, is minimized.

The exact solutions of MM and BP relate in the following way. It is possible to schedule all jobs in an MM instance on $m$ machines with makespan $C_{\max }$ if and only if it is possible to pack all the items in a BP instance, where the size of item $j$ is $p_{j} / C_{\max }$, in $m$ bins. The connection between the optimal solutions does not remain valid for approximations. In particular, BP has an asymptotic FPTAS (see chapter R-12) while MM does not. However, this connection can be used to develop a PTAS for MM.

Let $O P T_{B P}(I)$ be the number of bins in the optimal solution of $\mathrm{BP}$, and let $O P T_{M M}(I)=C_{\max }$ be an optimal solution for MM. Denote by $\frac{I}{d}$ the BP input in which all the values are divided by $d$. We already argued that:

$$
O P T_{B P}\left(\frac{I}{d}\right) \leq m \Leftrightarrow O P T_{M M}(I, m) \leq d
$$

We define a dual approximation scheme for BP. For input $I$, we seek a solution with at most $O P T_{B P}$ bins, where each bin is filled to capacity at most $1+\varepsilon$. In other words, we relax the bin capacity constraint by a factor of $1+\varepsilon$. Let $\operatorname{dual}_{\varepsilon}(I)$ be such an algorithm and $D U A L_{\varepsilon}(I)$ be the number of bins in the corresponding packing.

Theorem 1.4 If there exists a dual approximation algorithm for BP, then there is a PTAS for the minimum makespan problem. 


\section{Proof}

The PTAS performs a binary search to find $O P T_{M M}$. In order to bound the range in which the optimal makespan is searched, two lower bounds and one upper bound for this value are used. The lower bounds are the length of the longest job, and the load on each machine when the total load is perfectly balanced. That is, let $\operatorname{SIZE}(I, m)=\max \left\{\frac{1}{m} \sum p_{i}, p_{\max }\right\}$, then $O P T_{M M} \geq S I Z E(I, m)$. The upper bound uses the fact that the simple List Scheduling algorithm obtains a 2-approximation ratio [21], therefore $O P T_{M M} \leq$ $2 \operatorname{SIZE}(I, m)$.

Now it is possible to perform a binary search to find $O P T_{M M}$. Instead of checking directly whether $O P T_{M M}<d$, the algorithm checks whether $D U A L_{\varepsilon}\left(\frac{I}{d}\right)<m$.

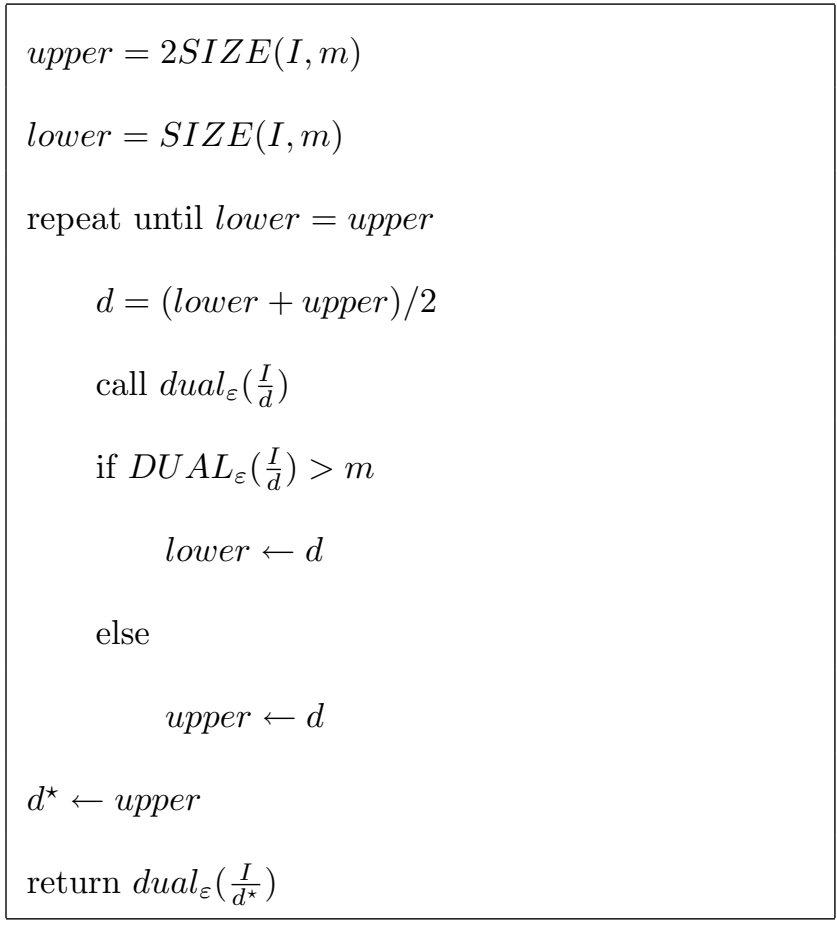

Initially, $O P T_{M M}(I, m) \leq$ upper $\Rightarrow O P T_{B P}\left(\frac{I}{\text { upper }}\right) \leq m$. Since $d u a l_{\varepsilon}$ is a relaxation of $B P, D U A L_{\varepsilon}\left(\frac{I}{u p p e r}\right) \leq$ $O P T_{B P}\left(\frac{I}{\text { upper }}\right)$. This implies that $D U A L_{\varepsilon}\left(\frac{I}{\text { upper }}\right) \leq m$. By the update rule, the above remains true during the execution of the loop. However,

$$
D U A L_{\varepsilon}\left(\frac{I}{\text { upper }}\right) \leq m \Rightarrow O P T_{M M}(I, m) \leq(1+\varepsilon) \text { upper }
$$

and thus $(1+\varepsilon)$ upper remains an upper bound on $\operatorname{OPT}_{M M}(I, m)$ during the search. Similarly, before the loop, $\operatorname{OPT}_{M M}(I, m) \geq$ lower, which remains true since $D U A L_{\varepsilon}\left(\frac{I}{\text { lower }}\right) \geq m$ is an invariant of the loop, 
and

$$
O P T_{B P}\left(\frac{I}{\text { lower }}\right) \geq D U A L_{\varepsilon}\left(\frac{I}{\text { lower }}\right) \geq m \Rightarrow O P T_{M M}(I, m) \geq \text { lower. }
$$

Thus, the solution value is bounded above by

$$
(1+\varepsilon) \cdot d^{\star}=(1+\varepsilon) \cdot \text { upper }=(1+\varepsilon) \cdot \text { lower } \leq(1+\varepsilon) O P T_{M M}(I, m)
$$

In practice, assume we stop the binary search after $k$ iterations. At this time, it is guaranteed that upper - lower $\leq 2^{-k} \operatorname{SIZE}(I, m) \leq 2^{-k} O P T_{M M}(I, m)$, and the value of the solution is bounded above by $(1+\varepsilon) \cdot d^{\star}=(1+\varepsilon) \cdot$ upper $\leq(1+\varepsilon) \cdot\left(\right.$ lower $\left.+2^{-k} O P T_{M M}(I, m)\right) \leq(1+\varepsilon)\left(1+2^{-k}\right) O P T_{M M}(I, m)$.

By choosing $k=O\left(\log \frac{1}{\epsilon}\right)$, we obtain a $\left(1+\frac{2}{\varepsilon}\right)$ approximation.

We will now describe the $d u a l_{\varepsilon}$ approximation scheme for bin packing. This scheme uses the rounding and grouping technique.

Theorem 1.5 There exists an $O\left(n^{\left\lceil\frac{1}{\varepsilon^{2}}\right\rceil}\right)$-time dual approximation scheme for bin packing.

\section{Proof}

Recall that for a give $\varepsilon$, the dual approximation algorithm needs to find a packing using at most $O P T_{B P}$ bins and each bin is packed with items whose total size is at most $1+\varepsilon$. The basic idea is to remove first all "small" items and then round the sizes of the "big" items in order to get an instance with a fixed number of distinct item sizes. This problem is solved exactly via a dynamic programming algorithm, and its solution induces a solution for the original instance where each bin is filled up to capacity $1+\varepsilon$.

The first observation is that small items, whose sizes are less that $\varepsilon$, can be initially omitted. The problem will be solved for big items only and the small items will be added later on greedily, in the following way: if there is a bin filled with items of total size less than 1, small items are added to it; otherwise, a new bin is opened. If no new bin is opened, then surely no more than the optimum number of bins is used (as the dual PTAS uses the minimal number of bins for the big items). If bins were added, then all original bins are filled to capacity at least 1 , and all the new bins (except maybe the last one) are also filled to capacity at least 1. This is optimal since $O P T \geq\left\lceil\sum p_{i}\right\rceil \geq D U A L_{\varepsilon}$. We conclude that, without loss of generality, all items are of size $\varepsilon \leq p_{i} \leq 1$. Divide this range into intervals of size $\varepsilon^{2}$. This yields $S=\left\lceil\frac{1}{\varepsilon^{2}}\right\rceil$ intervals. Denote by $l_{i}$ the end points of the intervals and define $b_{i}$ to be the number of elements with sizes in the interval $\left(l_{i}, l_{i+1}\right]$. 
We now examine a packed bin. Because the minimal item size is $\varepsilon$, there can be at most $\left\lfloor\frac{1}{\epsilon}\right\rfloor$ items in it. Denote by $X_{i}$ the number of items in the bin whose sizes are in the interval $\left(l_{i}, l_{i+1}\right] . X_{i}$ is in the range $\left[0,\left\lfloor\frac{1}{\epsilon}\right\rfloor\right)$. Define a vector $\left(X_{1}, \ldots, X_{S}\right)$ as the configuration of the bin. The number of feasible configurations is bounded above by $\left\lfloor\frac{1}{\epsilon}\right\rfloor$. A configuration is feasible if and only if $\sum_{i=1}^{S} X_{i} l_{i} \leq 1$.

For any bin $B$ whose packing forms a feasible configuration, it holds that the total size of the items in the bin is bounded by

$$
\sum_{j \in B} p_{j} \leq \sum_{j \in B} X_{j} l_{j+1} \leq \sum_{j \in B} X_{j}\left(l_{j}+\varepsilon^{2}\right) \leq 1+\varepsilon^{2} \sum_{j \in B} X_{j} \leq 1+\varepsilon^{2} \cdot \frac{1}{\epsilon} \leq 1+\varepsilon
$$

Therefore, it is sufficient to solve the instance with all item sizes rounded down to sizes in $\left\{l_{1}, \ldots, l_{S}\right\}$.

Finally, we describe a dynamic programming algorithm which solves the bin packing problem exactly when the number of distinct item sizes is fixed. Define $B I N S\left(b_{1}, b_{2}, \ldots, b_{S}\right)$ as the minimal number of bins required to pack $b_{1}$ items of size $l_{1}, b_{2}$ items of size $l_{2}, \ldots$, and $b_{S}$ items of size $l_{S}$. Let $\mathcal{C}$ denote the set of all feasible configurations. Observe that, by a standard dynamic programming argument,

$$
\operatorname{BINS}\left(b_{1}, b_{2}, \ldots, b_{S}\right)=1+\min _{\mathcal{C}} B I N S\left(b_{1}-X_{1}, b_{2}-X_{2}, \ldots, b_{S}-X_{S}\right)
$$

We minimize over all possible vectors $\left(X_{1}, X_{2}, \ldots, X_{s}\right)$ that represent a feasible packing of the "first" bin (counted by the constant 1), and the best way to pack the remaining items (this is the the recursive call). Thus, the dynamic programming build a table of size $n^{S}$, where the calculation of each entry requires $O\left(\left\lfloor\frac{1}{\epsilon}\right\rfloor^{S}\right)$

This yields a running time of

$$
O\left(n^{S} \cdot\left\lfloor\frac{1}{\epsilon}\right\rfloor^{S}\right)=O\left(\left(\frac{n}{\varepsilon}\right)^{\left\lceil\frac{1}{\varepsilon^{2}}\right\rceil}\right)=O\left(n^{\left\lceil\frac{1}{\varepsilon^{2}}\right\rceil}\right)
$$

The technique of applying enumeration to a compacted instance through grouping/rounding has been extensively used in PTASs for scheduling problems (see, e.g., [43], [28], [1]). A common approach for compacting the instance is to reduce the input parameters to poly-bounded, i.e., parameters whose values can be bounded as function of the input size. This approach is used, e.g. in the PTAS of Chekuri and Khanna for preemptive weighted flow time [13] (see also the survey paper [29]). 


\subsection{More on Grouping and Shifting}

In the following we outline two extensions of the techniques described in this section.

Randomized Grouping: In some cases, we need to define a partition of the input elements to groups $\left(I_{1}, \ldots, I_{k}\right)$, using for each element $x$ a parameter of the problem, $q(x)$, such that the elements in two groups $I_{j}$ and $I_{j+1}$ differ in their $q(x)$ value by roughly a factor of $\alpha$, for some $\alpha>1$. When such partition is infeasible, we can use randomization to achieve an expected separation between groups. For a parameter $\alpha>1$, the following randomized geometric grouping technique yields an expected separation that is logarithmic in $\alpha$. This technique extends the deterministic geometric rounding technique described in Section 2.2.2. Initially, pick a number $r \in[1, \alpha]$ at random, by a probability distribution having the density function $f(y)=1 / y \ln \alpha$. An element $x$ with the value $q(x)$ belongs to the group $I_{j}$ if $q(x) \in\left[r \alpha^{j}, r \alpha^{j+1}\right)$. Thus, the index of the group to which $x$ belongs, denoted $g(x)$, is a random variable which can take two possible values: $\left\lfloor\log _{\alpha} q(x)\right\rfloor$ or $\left\lfloor\log _{\alpha} q(x)\right\rfloor+1$. It can be shown that for a fixed $\alpha$, the number of distinct partitions induced by the random choices of $r$ is at most the number of elements in the input. This enables to easily derandomize algorithms that use randomized geometric grouping. The technique was applied, e.g., by Chekuri and Khanna [13] in a PTAS for preemptive weighted flow time.

Oblivious Shifting: While applying the standard shifting technique (as described in Section 2.2.2) requires knowing the initial input parameters, it is possible to apply shifting also when not all values are known apriori. In oblivious shifting, the input size is initially known, and the scheme starts by defining the number of values in the resulting instance, but the actual shifted values are revealed at a later stage, by optimizing on these values, considering the constraints of the problem. The technique can be used for defining a 'good' compacted instance from a partial solution for the problem, which can then enable to obtain a complete solution for the problem efficiently.

For example, the problem of bin packing with item fragmentation is solved in [46] in two steps. Given the input, we need to determine the set of items that will be fragmented, as well as the fragment sizes in a feasible approximate solution. Since the possible number of fragment sizes is large, a compact vector of fragments is generated, which contains a bounded number of unknown shifted fragment sizes. The actual 
sizes of the shifted fragments are determined by solving a linear program which attempts to find a feasible packing of these fragments. A detailed description is given in [46].

\section{Rounding Linear Programs}

In this section we discuss approximations obtained using linear programming relaxation of the integer program formulation of a given optimization problem. We refer the reader to Chapter R-5 and R-94 in this book for further background on linear programming and rounding linear programs. Most generally, the technique is based on solving a linear programming relaxation of the problem, for which an exact or approximate solution can be obtained efficiently. This solution is then rounded, thus yielding an approximate integral solution. The (fractional) solution obtained for the LP needs to have some nice properties that would allow rounding to be not too harmful, in terms of $\varepsilon$, the accuracy parameter of the scheme. One such property of a linear program which is commonly used is the existence of a small basic solution. We illustrate below the usage of this property, with examples from vector scheduling and covering integer programs. A linear program has a small basic solution, if there exists an optimal solution in which the number of non-zero variables is small as function of the input size and the accuracy parameter, $\varepsilon$. For such a solution, the error incurred by rounding can be bounded, such that the resulting integral solution is within factor of $1+\varepsilon$ from the optimal. A natural example is the class of linear programs in which either the number of variables or the number of constraints is some fixed constant. For such programs, there exists a basic solution in which the number of non-zero variables is fixed. however, depending on the problem, and in particular, on the value of an optimal solution for the LP, a basic solution can be 'small', even if the number of non-zero variables is relatively large, for example, $\Omega(\varepsilon n)$, where $n$ is the number of variables.

LP rounding can be combined with the techniques described in Section 2. In Section 3.1 we show the usage of LP rounding for a given subset of input elements satisfying certain properties. In Section 3.2 we show how LP rounding can be combined with the selection of all possible (small) subsets. 


\subsection{Solving LP for a Subset of Elements}

As mentioned in Section 2.1, in many problems, an approximation scheme can be obtained by partitioning a set of input elements to subsets, and solving the problem for each subset separately. For some subsets, a good solution can be obtained by rounding an LP relaxation of the problem.

In certain assignment problems, we can find an almost integral basic solution for an LP, for part of the input, since the relation between the number of variables and non-trivial constraints in the linear programming relaxation, combined with the assignment requirement of the problem, imply that only few variables can get fractional values. This essential property is used, e.g. in the PTAS of Chekuri and Khanna for the vector scheduling (VS) problem [11]. The VS problem is to schedule $d$-dimensional jobs on $m$ identical machines, such that the maximum load over all dimensions and over all machines is minimized. Formally, an instance $I$ of VS consists of $n$ jobs, $J_{1}, \ldots, J_{n}$, where $J_{j}$ is associated with a rational $d$-dimensional vector

$\left(p_{j}^{1}, \ldots, p_{j}^{d}\right)$, and $m$ machines. We need to assign the jobs to the machines, i.e., schedule a subset of the jobs $A_{i}$ on machine $i, 1 \leq i \leq m$, such that $\max _{1 \leq i \leq m} \max _{1 \leq h \leq d} \sum_{J_{j} \in A_{i}} p_{j}^{h}$ is minimized.

Note that in the special case where $d=1$ we get the multiprocessor scheduling problem. The PTAS of Chekuri and Khanna [11] for VS where $d$ is fixed applies a non-trivial generalization of the PTAS of Hochbaum and Shmoys for the case $d=1$ [23]. The scheme is based on a primal-dual approach, in which the primal problem is VS and the dual problem is vector packing. Thus, the machines are viewed as $d$ dimensional bins, and the schedule length — as bin capacity (or height). W.l.o.g., we may assume that the optimal schedule has the value 1 . Given an $\varepsilon>0$ and a correct guess of the optimal value, we describe below an algorithm $A_{\varepsilon}$ that returns a schedule of height at most $1+\varepsilon$. Arriving at correct guess involves a binary search for the optimal value (which can be done in polynomial time; see below).

Let $\delta=\varepsilon / d$ be a parameter. The scheme starts with a preprocessing step, which enables to bound the ratio of the largest coordinate to the smallest non-zero coordinate in any input vector. Specifically, let $\left\|J_{j}\right\|_{\infty}=\max _{1 \leq h \leq d} p_{j}^{h}$ be the $\ell_{\infty}$ norm of $J_{j}, 1 \leq j \leq n$, then, for any $J_{j}$, and any $1 \leq h \leq d$, if $p_{j}^{h} \leq \delta\left\|J_{j}\right\|_{\infty}$, we set $p_{j}^{h}=0$. As shown in [11], any valid schedule for the modified instance, $I^{\prime}$, yields a valid solution for the original instance, $I$, whose height is at most $(1+\varepsilon)$ times that of $I^{\prime}$.

We consider from now on only the transformed instances. The scheme proceeds by partitioning the jobs 
to the sets $L$ (large) and $S$ (small). The set $L$ consists of all vectors whose $\ell_{\infty}$ norm is greater than $\delta$, and $S$ contains the remaining vectors. The algorithm $A_{\varepsilon}$ packs first the large jobs and then the small jobs. Note, that while in the case of $d=1$ these packings are done independently, for $d \geq 2$, we need to consider the interaction between these two sets. Similar to the scheme of Hochbaum and Shmoys [23], a valid schedule is found for the jobs by guessing a configuration. In particular, let the $d$-tuple $\left(a_{1}, \ldots, a_{d}\right) 0 \leq a_{h} \leq\lceil 1 / \varepsilon\rceil$, $1 \leq h \leq d$, denote a capacity configuration, that is, the way some bin is filled. Since $d \geq 2$ is a constant, the possible number of capacity configurations, given by $W=(1+\lceil 1 / \varepsilon\rceil)^{d}$, is also a constant. Then, by numbering the capacity configurations, we describe by a $W-$ tuple $M=\left(m_{1}, \ldots, m_{W}\right)$ the number of bins having capacity configuration $w$, where $1 \leq w \leq W$. The possible number of bin configurations is then $O\left(m^{W}\right)$. This allows to guess a bin configuration which yields the desired $(1+\varepsilon)$-approximate solution in polynomial time.

We say that a packing of vectors in a bin respects a capacity configuration $\left(a_{1}, \ldots, a_{d}\right)$ if the height of the packing is smaller than $\varepsilon a_{h}$ for any $1 \leq h \leq d$. Given a capacity configuration $\left(a_{1}, \ldots, a_{d}\right)$, we define the empty capacity configuration to be the $d$-tuple $\left(\bar{a}_{1}, \ldots, \bar{a}_{d}\right)$, where $\bar{a}_{h}=\lceil 1 / \varepsilon\rceil+1-a_{h}$, for $1 \leq h \leq d$. For a given bin configuration $M$, we denote by $\bar{M}$ the bin configuration obtained by taking for each of the bins in $M$ the corresponding empty capacity configuration.

The scheme performs the following two steps for each possible bin configuration, $M$ : $(i)$ decides whether vectors in $L$ can be packed respecting $M$, and (ii) decides whether vectors in $S$ can be packed respecting $\bar{M}$. Given that we have guessed the correct bin configuration $M$, both steps will succeed, and we get a packing of height at most $1+\varepsilon$.

We now describe how the scheme packs the large and the small vectors. The vectors in $L$ are packed using rounding and dynamic programming. In particular, since by definition, any entry in a vector in $L$ has the value $\delta^{2}$ or larger, we use geometric rounding, that is, for each vector $J_{j}$, and any entry $p_{j}^{h}, 1 \leq h \leq d$, we round down $p_{j}^{h}$ to the nearest value of the form $\delta^{2}(1+\varepsilon)^{t}$, for $0 \leq t \leq\left\lceil\frac{2}{\varepsilon} \log 1 / \delta\right\rceil$. Denote the resulting set of vectors $L^{\prime}$, and the modified instance $I^{\prime}$. The vectors in $L^{\prime}$ can be partitioned into

$$
q=\left(1+\left\lceil\frac{2}{\varepsilon} \log 1 / \delta\right\rceil\right)^{d}
$$

classes. The proofs of the next lemmas are given in [11]. 
Lemma 1.1 Given a solution for $I^{\prime}$, replacing each vector in $L^{\prime}$ by the corresponding vector in $L$ results in a valid solution for $I$ whose height is at most $1+\varepsilon$ times that of $I^{\prime}$.

Lemma 1.2 Given a correct guess of a bin configuration $M$, there exists an algorithm which finds a packing of the vectors in $L^{\prime}$ that respects $M$, and whose running time is $O\left((d / \delta)^{q} m n^{q}\right)$, where $q$ is given in (1.1).

The small vectors are packed using a linear programming relaxation and careful rounding. Renumber the vectors in $S$ by $1, \ldots,|S|$. Let $x_{j i} \in\{0,1\}$ be an indicator variable for the assignment of the vector $J_{j}$ to machine $i, 1 \leq j \leq n, 1 \leq i \leq m$. In the LP relaxation $x_{j i} \geq 0$. We solve the following linear program.

$$
\begin{array}{cl}
(L P) \quad \sum_{J_{j} \in S} p_{j}^{h} x_{j i} \leq b_{i}^{h} & 1 \leq i \leq m, \quad 1 \leq h \leq d \\
\sum_{i=1}^{m} x_{j i}=1 & 1 \leq j \leq|S| \\
x_{j i} \geq 0 & 1 \leq j \leq n, \quad 1 \leq i \leq m
\end{array}
$$

The constraints (1.2) guarantee that the packing does not exceed a given height bound in any dimension (i.e., the available height after packing the large vectors). The constraints (1.3) reflect the requirement that each vector is assigned to one machine. A key property of the LP, which enables to obtain an integral solution that is close to the fractional, is given in the next result.

Lemma 1.3 In any basic feasible solution for $L P$, at most $d \cdot m$ vectors are assigned (fractionally) to more than one machine.

\section{Proof}

Recall that the number of non-zero variables, in any basic solution for a linear program, is bounded by the number of tight constraints in some optimal solution (since non-tight constraints can be omitted). Since the number of non-trivial constraints (i.e., constraints other than $\left.x_{j i} \geq 0\right)$ is $(|S|+d \cdot m$ ), it follows that the number of strictly positive variables in any basic solution is at most $(|S|+d \cdot m)$. Since each vector is assigned to at least one machine, the number of vectors which are fractionally assigned to more than one machine is at most $d \cdot m$.

The above type of argument was first made and exploited by Potts [39] in the context of parallel machine scheduling. It was later applied for other problems, such as job shop scheduling (see e.g., [27]). 
Thus, we solve the above program and obtain a basic solution. Denote by $S^{\prime}$ the set of vectors which are assigned fractionally to two machines or more. Since $\left|S^{\prime}\right| \leq d \cdot m$, we can partition the set $S^{\prime}$ to subsets of size at most $d$ each, and schedule the $i$-th set to the $i$-th machine. Since $\left\|J_{j}\right\|_{\infty} \leq \delta=\varepsilon / d$, for all $J_{j} \in S^{\prime}$, the total height of the machines is violated at most by $\varepsilon$ in any dimension. We can therefore summarize in the following theorem.

Theorem 1.6 For any $\varepsilon>0$, there is a $(1+\varepsilon)$-approximation algorithm for VS whose running time is $(n d / \varepsilon)^{O(f)}$, where $f=O\left(\left(\frac{\ln (d / \varepsilon)}{\varepsilon}\right)^{d}\right)$.

\section{Proof}

By the above discussion, given the correct guess of the optimal value, the scheme yields a schedule of value (height) at most $1+O(\varepsilon)$ the optimal. We need to find a packing of the vectors in $L$ and $S$, for each bin configuration $M$. The running time for a single configuration is dominated by the packing of $L$, and since the number of configurations is $m^{W}=O\left(n^{O\left(1 / \varepsilon^{d}\right)}\right)$, we get the running time from Lemma 1.2. The value of an optimal schedule can be guessed, within factor $1+\varepsilon$, by obtaining first a $(d+1)$-approximate solution. This can be done by applying an approximation algorithm for resource constrained scheduling due to [20].

\subsection{LP Rounding Combined with Enumeration}

As described in Section 2.1, a common technique for obtaining a PTAS is to extend all possible solutions for small subsets of elements. This technique can be combined with LP rounding as follows. Repeatedly select a small subset of input elements, $S_{g} \subseteq I$, to be the basis for an approximate solution; solve an LP for the remaining elements, $I \backslash S_{g}$. Select the subset $S_{g}$ which gives the best solution. We exemplify the usage of the technique to obtain a PTAS for covering integer programs with multiplicity constraints (CIP). In this core problem, we must fill up an $R$-dimensional bin by selecting (with bounded number of repetitions) from a set of $n R$-dimensional items, such that the overall cost is minimized. Formally, let $A=\left\{a_{j i}\right\}$ denote the sizes of the items in the $R$ dimensions, $1 \leq j \leq R, 1 \leq i \leq n$; the cost of item $i$ is $c_{i} \geq 0$. Let $x_{i}$ denote the number of copies selected from item $i, 1 \leq i \leq n$. We seek an $n$-vector $\mathbf{x}$ of non-negative integers, which minimizes $c^{T} \mathbf{x}$, subject to the $R$ constraints given by $A \mathbf{x} \geq b$, where $b_{j} \geq 0$ is the size of the bin in dimension 
$j$. In addition, we have multiplicity constraints for the vector $\mathbf{x}$, given by $\mathbf{x} \leq d$, where $d \in\{1,2, \ldots\}^{n}$.

Covering integer programs form a large subclass of integer programs encompassing such NP-hard problems as minimum knapsack and set cover. This implies the hardness of CIP in fixed dimension (i.e., where $R$ is a fixed constant). For general instances, the hardness of approximation results for set cover carry over to CIP. Comprehensive surveys of known results for CIP and $C I P_{\infty}$, where the multiplicity constraints are omitted, are given in [44] and in [35] (see also in [36]).

We now describe a PTAS for CIP in fixed dimension. The scheme presented in [44] builds on the classic LP-based scheme due to Frieze and Clarke for the R-dimensional knapsack problem [17]. Consider an instance of CIP in fixed dimension, $R$. We want to minimize $\sum_{i=1}^{n} c_{i} x_{i}$ subject to the constraints $\sum_{i=1}^{n} a_{i j} x_{i} \geq b_{j}$ for $j=1, \ldots, R$, and $x_{i} \in\left\{0,1, \ldots d_{i}\right\}$ for $i=1 \ldots, n$.

Assume that we know the optimal cost, $C$, for the CIP instance. The scheme of [44] uses a reduction to the binary minimum $R$-dimensional multiple choice knapsack ( $R$-MMCK) problem. For some $R \geq 1$, an instance of binary R-MMCK consists of a single $R$-dimensional knapsack, of size $b_{j}$ in the $j$-th dimension, and $m$ sets of items. Each item has an $R$-dimensional size and is associated with a cost. The goal is to pack a subset of items, by selecting at most one item from each set, such that the total size of the packed items in dimension $j$ is at least $b_{j}, 1 \leq j \leq R$, and the overall cost is minimized.

Given the value of $C$, the parameter $\varepsilon$ and a CIP instance with bounded multiplicity, we construct an R-MMCK instance in which the knapsack capacities in the $R$ dimensions are $b_{j}, 1 \leq j \leq R$. Also, we have $n$ sets of items denoted by $A^{i}, 1 \leq i \leq n$. Let $\hat{K}^{i}$ be the value satisfying $d_{i} c_{i} \in\left[\hat{K}^{i} \varepsilon C / n,\left(\hat{K}^{i}+1\right) \varepsilon C / n\right)$, then the number of items in $A^{i}$ is $K^{i}=\min \left(\hat{K}^{i},\lfloor n / \varepsilon\rfloor\right)$. The set $A^{i}$ represents all possible values which $x_{i}$ can take in the solution for CIP. In particular, the $k$-th item in $A^{i}$, denoted $(i, k)$, represents the assignment of a value in $\left[0, d_{i}\right]$ to $x_{i}$, such that $c(i, k)$, the total cost incurred by item $i$ is in $[k \varepsilon C / n,(k+1) \varepsilon C / n)$. This total cost is rounded down to the nearest integral multiple of $\varepsilon C / n$; thus, $c(i, k)=k \varepsilon C / n$. The size of the item $(i, k)$ in dimension $j, 1 \leq j \leq R$, is given by $s_{j}(i, k)=a_{i j}$.

Given an instance of R-MMCK, we guess a partial solution, given by a small size set, $S$; these items have the maximal costs in some optimal solution. The size of $S$ is a fixed constant, namely, $|S|=h=\left\lfloor\frac{2 R(1+\varepsilon)}{\varepsilon}\right\rfloor$. The set $S$ will be extended to an approximate solution, by solving a linear program for the remaining items. 
We choose the value of $h$ such that the resulting solution is guaranteed to be within $1+\varepsilon$ from the optimal, as computed below. Let $E(S)$ be the subset of items with costs that are larger than the minimal cost of any item in $S$, that is, $E(S)=\left\{(i, k) \notin S \mid c(i, k)>c_{\min }(S)\right\}$, where $c_{\min }(S)=\min _{(i, k) \in S} c(i, k)$. We select all the items $(i, k) \in S$, and eliminate from the instance all the items $(i, k) \in E(S)$ and the sets $A^{i}$ from which an item has been selected. In the next step we find an optimal basic solution for the following linear program, in which $x_{i, k}$ is an indicator variable for the selection of the item $(i, k)$.

$$
\begin{aligned}
(L P(S)) \quad \text { minimize } \quad & \sum_{i=1}^{n} \sum_{k=1}^{K^{i}} x_{i, k} \cdot c(i, k) \\
\text { subject to }: \quad & \sum_{k=1}^{K^{i}} x_{i, k} \leq 1 \text { for } i=1, \ldots, n, \\
& \sum_{i=1}^{n} \sum_{k=1}^{K^{i}} s_{j}(i, k) x_{i, k} \geq n_{j} \text { for } j=1, \ldots, R \\
& 0 \leq x_{i, k} \leq 1 \text { for }(i, k) \notin S \cup E(S) \\
& x_{i, k}=1 \text { for }(i, k) \in S \\
& x_{i, k}=0 \text { for }(i, k) \in E(S)
\end{aligned}
$$

Given an optimal fractional solution for the above program, we get an integral solution as follows. For any $i, 1 \leq i \leq n$, let $k_{\max }=k_{\max }(i)$ be the maximal value of $1 \leq k \leq K^{i}$ such that $x_{i, k}>0$, then we set $x_{i, k_{\max }}=1$ and, for any other item in $A^{i}, x_{i, k}=0$. Finally, we return to the CIP instance and assign to $x_{i}$ the maximum value for which the total (rounded down) cost for item $i$ is $c\left(i, k_{\max }\right)$.

The next three lemmas show that the scheme yields a $(1+\varepsilon)$-approximation to the optimal cost, and that the resulting integral solution is feasible.

Lemma 1.4 If there exists an optimal (integral) solution for CIP with cost $C$, then the integral solution obtained from the rounding for $R$-MMCK has the cost $\hat{z} \leq(1+\varepsilon) C$.

\section{Proof}

Let $\mathbf{x}^{*}$ be an optimal (fractional) solution for the linear program $\operatorname{LP}(S)$, and let $S^{*}$ be the corresponding subset of items, that is, $S^{*}=\left\{(i, k) \mid x_{i, k}^{*}=1\right\}$. If $\left|S^{*}\right|<h$ then we are done: in some iteration, the scheme with try $S^{*}$; otherwise, let $S^{*}=\left\{\left(i_{1}, k_{1}\right), \ldots,\left(i_{g}, k_{g}\right)\right\}$, such that $c\left(i_{1}, k_{1}\right) \geq \cdots \geq c\left(i_{g}, k_{g}\right)$, for some $g>h$. 
Let $S_{h}^{*}=\left\{\left(i_{1}, k_{1}\right), \ldots,\left(i_{h}, k_{h}\right)\right\}$, and $\sigma=\sum_{t=1}^{h} c\left(i_{t}, k_{t}\right)$. Then, for any item $(i, k) \notin\left(S_{h}^{*} \cup E\left(S_{h}^{*}\right)\right)$, we have $c(i, k) \leq \sigma / h$. Let $z^{*}, \hat{z}$ denote the optimal (integral) solution and the solution output by the scheme for the R-MMCK instance, respectively. We denote by $\mathbf{x}^{B}\left(S_{h}^{*}\right), \mathbf{x}^{I}\left(S_{h}^{*}\right)$ the basic and integral solutions of LP(S) as computed by the scheme, for the initial guess $S_{h}^{*}$.

By the above rounding method, for any $1 \leq i \leq n$, the cost of the item selected from $A^{i}$ is $c\left(i, k_{\max }\right)$. Let $F$ denote the set of items for which the basic variable was a fraction, that is, $F=\left\{(i, k) \mid x_{i, k}^{B}\left(S_{h}^{*}\right)<1\right\}$.

Then, we get that

$$
\begin{aligned}
z^{*} & \geq \sum_{i=1}^{n} \sum_{k=1}^{K^{i}} c(i, k) x_{i, k}^{B}\left(S_{h}^{*}\right) \\
& \geq \sum_{i=1}^{n} \sum_{k=1}^{K^{i}} c(i, k) x_{i, k}^{I}\left(S_{h}^{*}\right)-\delta
\end{aligned}
$$

where $\delta=\sum_{(i, k) \in F} c(i, k)$, and $F$ is the set of items for which the basic variable was a fraction, that is, $F=\left\{(i k) \mid x_{i, k}^{B}\left(S_{h}^{*}\right)<1\right\}$.

Recall that in any basic solution for a linear program, the number of non-zero variables is bounded by the number of tight constraints in some optimal solution. Assume that in the optimal (fractional) solution of $L P\left(S_{h}^{*}\right)$ there are $L$ tight constraints, where $0 \leq L \leq n+R$. Then in the basic solution $\mathbf{x}^{B}\left(S_{h}^{*}\right)$, at most $L$ variables can be strictly positive. Thus, at least $L-2 R$ variables get an integral value (i.e. ' 1 '), and $|F| \leq 2 R$. Note that, for any $(i, k) \in F, c(i, k) \leq \sigma / h$, since $F \cap\left(S_{h}^{*} \cup E\left(S_{h}^{*}\right)\right)=\emptyset$. Hence, we get that $z^{*} \geq \hat{z}+\frac{2 R \sigma}{h} \geq \hat{z}+\frac{2 R \hat{z}}{h} \geq \frac{\hat{z}}{1+\varepsilon}$.

Lemma 1.5 The scheme yields a feasible solution for the CIP instance.

\section{Proof}

The feasibility of the solution follows from the definition of $K^{i}$ and the fact that $k_{\max }(i) \leq K^{i}$. Thus, the value assigned to $x_{i}$ is at most $d_{i}$.

Lemma 1.6 The cost of the integral solution for the CIP instance is at most $\hat{z}+\varepsilon C$.

\section{Proof}

We note that, for any variable $x_{i}$, the cost incurred by the integral solution for the CIP instance is at most 
$c\left(i, k_{\max }(i)\right)+\varepsilon C / n$. It follows that the total cost value for the R-MMCK instance is increased at most by $\varepsilon C$.

Note that $C$ can be guessed in polynomial time within factor $(1+\varepsilon)$, using binary search over the range $\left(0, \sum_{i=1}^{n} d_{i} c_{i}\right)$. Thus, combining the above lemmas we get:

Theorem 1.7 There is a polynomial time approximation scheme for CIP in fixed dimension.

Consider now the special case where the multiplicity constraints are omitted; that is, each variable $x_{i}$ can get any non-negative (integral) value. For this special case, we can use a linear programming formulation in which the number of constraints is $R$, which is fixed. A PTAS for this problem can be derived from the scheme of Chandra et al. [10] for integer multidimensional knapsack. drawing from recent results on solutions of CIPs, we describe below the PTAS of [44], that achieves a better running time, by using a fast approximation scheme for solving the linear program.

A Scheme for $C I P_{\infty}$ The scheme, called below multi-dimensional cover with parameter $\varepsilon\left(M D C_{\varepsilon}\right)$, proceeds in the following steps.

(i) For a given $\varepsilon \in(0,1)$, let $\delta=\lceil R \cdot((1 / \varepsilon)-1)\rceil$.

(ii) We renumber the items by $1, \ldots, n$, such that $c_{1} \geq c_{2} \geq \cdots \geq c_{n}$.

(iii) Denote by $\Omega$ the set of integer vectors $\mathbf{x}=\left(x_{1}, \ldots, x_{n}\right)$ satisfying $x_{i} \geq 0$ and $\sum_{i=1}^{n} x_{i} \leq \delta$. For any vector $\mathbf{x} \in \Omega$ : Let $d \geq 1$ be the maximal integer $i$ for which $x_{i} \neq 0$. Find a $(1+\varepsilon)$-approximation to the optimal (fractional) solution of the following linear program.

$$
\begin{aligned}
\left(L P^{\prime}\right) \quad \text { minimize } & \sum_{i=d+1}^{n} c_{i} z_{i} \\
\text { subject to }: \quad & \sum_{i=d+1}^{n} a_{i j} z_{i} \geq b_{j}-\sum_{i=1}^{n} a_{i j} x_{i} \text { for } j=1, \ldots, R \\
& z_{i} \geq 0, \text { for } i=d+1, \ldots, n
\end{aligned}
$$

The constraints (1.6) reflect the fact that we need to fill in each dimension $j$ at least the capacity $b_{j}-$ $\sum_{i=1}^{n} a_{i j} x_{i}$, once we obtained the vector $\mathbf{x}$. 
Let $\hat{z}_{i}, d+1 \leq i \leq n$ be a $(1+\varepsilon)$-approximate solution for $L P^{\prime}$. We take $\left\lceil\hat{z}_{i}\right\rceil$ as the integral solution. Denote by $C_{M D C}(\mathbf{x})=\sum_{i=d+1}^{n} c_{i}\left\lceil\hat{z}_{i}\right\rceil$ the value obtained from the rounded solution, and let $c(\mathbf{x})=\sum_{i=1}^{n} c_{i} x_{i}$.

(iv) Select the vector $\mathbf{x}$ for which $C_{M D C_{\varepsilon}}(\mathbf{x})=\min _{\mathbf{x}}\left(c(\mathbf{x})+C_{M D C}(\mathbf{x})\right)$.

We now show that $M D C_{\varepsilon}$ is a PTAS for $C I P_{\infty}$. Let $C_{o}$ be the cost of an optimal integral solution for the $C I P_{\infty}$ instance.

Theorem $1.8 M D C_{\varepsilon}$ is a PTAS for CIP $P_{\infty}$ which satisfies the following. (i) If $C_{o} \neq 0, \infty$ then $C_{M D C_{\varepsilon}} / C_{o}<$ $1+\varepsilon$. (ii) The running time of algorithm $M D C_{\varepsilon}$ is $O\left(n^{\lceil R / \varepsilon\rceil} \cdot \frac{1}{\varepsilon^{2}} \log C\right)$, where $C=\max _{1 \leq i \leq n} c_{i}$ is the $\max$ imal cost of any item, and its space complexity is $O(n)$.

We use in the proof the next lemma.

Lemma 1.7 For any $\varepsilon>0$, a $(1+\varepsilon)$-approximation to the optimal solution for $L P^{\prime}$ can be found in $O\left(1 / \varepsilon^{2} R \log (C \cdot R)\right)$ steps.

\section{Proof}

For a system of inequalities as given in $L P^{\prime}$, there is a solution in which at most $R$ variables get non-zero values. This follows from the fact that the number of non-trivial constraints is $R$. Hence, it suffices to solve $L P^{\prime}$ for the $\left(\begin{array}{c}n-d \\ R\end{array}\right)$ possible subsets of $R$ variables, out of $\left(z_{d+1}, \ldots, z_{n}\right)$. This can be done in polynomial time since $R$ is fixed. Now, for each subset of $R$ variables we have an instance of the fractional covering problem, for which we can use a fast approximation scheme (see, e.g., in [16]) to obtain a $(1+\varepsilon)$-approximate solution.

Proof of Theorem 1.8: For showing $(i)$, assume that the optimal (integral) solution for the $C I P_{\infty}$ instance is obtained by the vector $\mathbf{y}=\left(y_{1}, \ldots, y_{n}\right)$. If $\sum_{i=1}^{n} y_{i} \leq \delta$ then $C_{M D C_{\varepsilon}}=C_{o}$, since in this case $\mathbf{y}$ is a valid solution, and $\mathbf{y} \in \Omega$, therefore, in some iteration $M D C_{\varepsilon}$ will examine $\mathbf{y}$. Suppose that $\sum_{i=1}^{n} y_{i}>\delta$, then we define the vector $\mathbf{x}=\left(y_{1}, \ldots, y_{d-1}, x_{d}, 0, \ldots, 0\right)$, such that $y_{1}+\cdots+y_{d-1}+x_{d}=\delta$. (Note that $x_{d} \neq 0$.) Let $\tilde{C}_{o}(\mathbf{x})=\sum_{i=d+1}^{n} c_{i} \hat{z}_{i}$ be the approximate fractional solution for $L P^{\prime}$. We have that $\mathbf{x} \in \Omega$, therefore

$$
C_{M D C}(\mathbf{x})-\tilde{C}_{o}(\mathbf{x}) \leq R c_{d}
$$

Let $C_{o}(\mathbf{x})$ be the optimal fractional solution for $L P^{\prime}$ with the vector $\mathbf{x}$. Note that $C_{o}$, the optimal (integral) solution for $C I P_{\infty}$, satisfies $C_{o}>c(\mathbf{x})+C_{o}(\mathbf{x})$, since $C_{o}(\mathbf{x})$ is a lower bound for the cost incurred by the 
integral values $y_{d+1}, \ldots, y_{n}$. In addition, $c(\mathbf{x})+C_{M D C}(\mathbf{x}) \geq C_{M D C_{\varepsilon}}$. Hence, we get that

$$
\begin{aligned}
\frac{C_{o}}{C_{M D C_{\varepsilon}}} & \geq \frac{c(\mathbf{x})+C_{o}(\mathbf{x})}{c(\mathbf{x})+C_{M D C}(\mathbf{x})}>1-\frac{C_{M D C}(\mathbf{x})-C_{o}(\mathbf{x})}{c(\mathbf{x})+C_{M D C}(\mathbf{x})-C_{o}(\mathbf{x})} \\
& \geq 1-\frac{C_{M D C}(\mathbf{x})-\tilde{C}_{o}(\mathbf{x})(1-\varepsilon)}{c(\mathbf{x})+C_{M D C}(\mathbf{x})-\tilde{C}_{o}(\mathbf{x})} \\
& \geq(1-\varepsilon)\left(1-\frac{C_{M D C}(\mathbf{x})-\tilde{C}_{o}(\mathbf{x})}{c(\mathbf{x})+C_{M D C}(\mathbf{x})-\tilde{C}_{o}(\mathbf{x})}\right) \\
& \geq(1-\varepsilon)\left(1-\frac{C_{M D C}(\mathbf{x})-\tilde{C}_{o}(\mathbf{x})}{\delta c_{d}+C_{M D C}(\mathbf{x})-\tilde{C}_{o}(\mathbf{x})}\right)
\end{aligned}
$$

The third inequality follows from the fact that $\tilde{C}_{o}(\mathbf{x}) \geq C_{o}(\mathbf{x}) \geq \tilde{C}_{o}(\mathbf{x})(1-\varepsilon)$, and the last inequality follows from the fact that $c(\mathbf{x}) \geq \delta c_{d}$.

Using (1.7), we get that $\frac{C_{o}}{C_{M D C_{\varepsilon}}} \geq(1-\varepsilon) 1-R c_{d} /\left(\delta c_{d}+R c_{d}\right) \geq(1-\varepsilon)^{2}$. By taking in the scheme $\tilde{\varepsilon}=\varepsilon / 2$ we get the statement in $(i)$.

Next, we show (ii). Note that $|\Omega|=O\left(n^{\delta}\right)$, since the number of possible choices of $n$ non-negative integers, whose sum is at most $\delta$ is bounded by $\left(\begin{array}{c}n+\delta \\ \delta\end{array}\right)$. Now, given a vector $\mathbf{x} \in \Omega$, we can compute $C_{M D C}(\mathbf{x})$ in $O\left(n^{R}\right)$ steps, since at most $R$ variables out of $z_{d+1}, \ldots, z_{n}$ can have non-zero values. Multiplying by the complexity of the FPTAS for fractional covering, as given in Lemma 1.7, we get the statement of the theorem.

Enumeration is combined with LP rounding in the PTAS of Chekuri and Khanna for Multiple Knapsack [12], as well as in the PTAS of Caprara et al. [9] for the knapsack problem with cardinalities constraints. The scheme of [9] is based on the scheme of Frieze and Clarke [17], with the running time improved by factor of $n$, the number of items. The scheme of [17] is the basis also for PTASs for other variants of the knapsack problem. (A comprehensive survey is given in [31]; see also in [45].)

\section{Approximation Schemes for Geometric Problems}

In this Section we present approximation techniques that are specialized for geometric optimization problems. For a complete description of these techniques we refer the reader to the survey by Arora [3], Chapter 11 in [47], and Chapters 8 and 9.3.3 in [24]. A typical input to a geometric problem is a set of elements in the 
space (such as points in the plane); the goal is to connect or pack these elements in a way that minimizes the resources used (e.g., total length of connecting lines, total number of covering objects).

\subsection{Randomized Dissection}

We present below the techniques used in the PTAS of Arora [2] for the Euclidean Traveling Salesman Problem (TSP). In the classical TSP problem, given non-negative edge weights for the complete graph $K_{n}$, the goal is to find a tour of minimum cost, where a tour refers to a cycle of length $n$. In other words, the goal is go find an ordering of the nodes such that the total cost of the edges along the path visiting all nodes according to this ordering is minimal. In general, TSP is NP-hard in the strong sense, and it can not be approximated within a multiplicative factor unless $\mathrm{P}=\mathrm{NP}$. Arora's PTAS considers the relaxed problem of Euclidean TSP: The input is $n$ points in $R^{d}$, and the edge weights are the Euclidean $\left(\ell_{2}\right)$ distances between them.

The idea of the PTAS is to dissect the plane into squares, and to look (using dynamic programming) for a tour that crosses the resulting grid lines only at specific points, denoted portals. The parameter $\varepsilon$ of the PTAS determines the depth of the recursive dissection as well as the density of the portals. A smaller $\varepsilon$ results in more portals and a finer dissection, that leads to a less restricted tour and a larger dynamic programming instance. Randomization is used to determine an initial shift of the grid lines.

A dissection of a square is a recursive partitioning into squares. It can be viewed as a tree of squares whose root is the square we started with. Each square in the tree is partitioned into four equal squares, which are its children. The leaves are squares of a small sidelength - determined by the parameter $\varepsilon$ of the PTAS.

The location of the grid lines is determined randomly as follows: Given a set of $n$ points in $R^{2}$, enclose the points in a minimum bounding square. Let $\ell$ be the side of this square. Let $p \in R^{2}$ be the lower left endpoint of the bounding box. Enclose the bounding box inside a larger square, denoted the enclosing box of sidelength $L=2 \ell$ and position the enclosing box such that $p$ has distance $a$ from the left edge and $b$ from the lower edge, where $a, b \leq \ell$ are chosen randomly. The randomized dissection is the dissection of this enclosing box. Note that the randomness is used only to determine the placement of the enclosing box (and its accompanying dissection). 
We will now describe Arora's PTAS for the Euclidean TSP problem that uses the above randomized dissection. Formally, for every $\varepsilon>0$, this PTAS finds a $(1+\varepsilon)$-approximation to Euclidean TSP.

First, perform randomized dissection to the bounding box of the $n$ points. Recall that $L$ is the side of the enclosing box. The recursive procedure of subdividing the squares stops when the side lengths of the squares becomes less than $L \varepsilon / 8 n$, or when each square at the last level contains at most one point. We may assume by scaling that $L$ is a power of 2 and that the sides of squares at the last level are unit length. Thus, at most $\log L$ iterations are required, and $L \leq 8 n / \varepsilon$. When there is more than one point in a unit square, consolidate them into one new "bigger" point. Any tour for the resulting set of points can be augmented to a tour for the original set of points with an increase in length bounded by $\sqrt{2} n L \varepsilon / 8 n$, which is negligible, since $L \leq O P T / 2$. Henceforth we shall assume there is at most one point per unit square.

The level of a square in the dissection is its depth from the root; the root square has level 0 . We also assign a level from 0 to $\log (L-1)$ to each horizontal and vertical grid line that participates in the dissection. The horizontal (resp., vertical) line that divides the enclosing box into two has level 0. Similarly, the $2^{i}$ horizontal and $2^{i}$ vertical lines that divide the level $i$ squares into level $i+1$ squares each have level $i$. The following property of a randomized dissection is used: Any fixed vertical grid line that intersects the bounding box of the instance has probability $\frac{2^{i}}{\ell}=\frac{2^{i+1}}{L}$ to be a line at level $i$.

Next, the location of the portals is determined: Let $m=\frac{1}{\varepsilon} \log L$. The parameter $m$ is the portal parameter that determines the density of the points the path can pass through. A level $i$ line has $2^{i+1} m$ equally spaced portals. In addition, we also refer to the corners of each a square as a portal. Since the level $i$ line has $2^{i+1}$ level $i+1$ squares touching it, we conclude that each side of the square has at most $m+2$ portals ( $m$ usual portals, and the 2 corners), and a total of at most $4 m+4$ portals on its boundary. A portal-respecting tour is one that, whenever it crosses a grid line, does so at a portal.

Finally, dynamic programming is used to find the optimum portal-respecting tour in time $2^{O(m)} L \log L$. Since $m=O(\log n / \varepsilon)$ we get a total running time of $n^{O(1 / \varepsilon)}$. The dynamic programming as well as the complete analysis of bounding the PTAS error and the time complexity are given in [3].

Note that since the PTAS uses randomization, its error of the PTAS is a random variable. Formally, let Let $O P T$ denote the cost of the optimum salesman tour and $O P T_{a, b, m}$ denote the cost of the best 
portal-respecting tour when the portal parameter is $m$ and the random shifts are $a, b$, then,

Theorem 1.9 The expectation (over the choice of $a, b$ ) of $O P T_{a, b, m}-O P T$ is at most $2 \log L / m O P T$, where $L$ is the sidelength of the enclosing box.

As listed in Arora's survey [3], this method of dissection can be used to develop PTASs for other geometric optimization problems such as minimum Steiner tree, facility location with capacities and demands, and Euclidean min-cost $k$-connected subgraph.

Another class of geometric optimization problem is the class of clustering problems, such as metric maxcut and $k$-median, In recent research on clustering problems the core idea in the design of an approximation scheme is to use sampling of data points at random from a biased distribution that depends on the pairwise distances. This technique is used e.g. in the PTAS of Fernandez de la Vega and Kenyon's for metric max-cut [19], and in Indyk's work for metric 2-clustering [26]. For more details on this technique and its usage the reader is referred to [18].

\subsection{Shifted Plane Partitions}

The next technique is called shifting: it is based on selecting the best solution over a (polynomial size) set of feasible solutions. Each candidate feasible solution is a result of a divide-and-conquer approach, in which the plane is partitioned into disjoint areas (strips). This technique fits geometric problems such as square packing or covering with disks. The above geometric problems arise in VLSI design, image processing and many other important applications. A common goal in these problems is to cover or pack elements (like points in the plane) into a minimal number of objects (like squares of given size).

Recall that each candidate solution is a result of a divide-and-conquer approach in which the plane is partitioned into disjoint areas (strips). A solution to the whole problem is formed by a union of the strips' solutions. Consequent solutions refer to consequent partitions of the plane into strips - that differ from each other by shifting the partitioning bars by the shifting parameter. The smaller the shifting parameter is, the more candidate solutions to be considered and the better resulting approximation.

We illustrate the shifting technique for the problem of covering $n$ points in the 2-dimensional plane. The complete analysis is given in $[24,25]$. Assume that the $n$ points are enclosed in an area $I$. The goal is to 
cover these points with a minimal number of disks of diameter $D$. Denote by $\ell$ the shifting parameter. The area $I$ is divided into vertical strips of width $D$. Each set of $\ell$ consequent strips are grouped together to form strips of width $\ell D$. Note that there are $\ell$ different ways to determine this grouping - and they can derive from each other by shifting the partitioning bars to the right over distance $D$. Denote the $\ell$ distinct partitions that results by $S_{1}, S_{2}, \ldots, S_{\ell}$.

Let $A$ be an algorithm to solve the covering problem on strips of width at most $\ell D$. The algorithm $A$ can be used to generate a solution for a given partition $S_{j}$ by applying $A$ to each strip in $S_{j}$ and union the disks used. The shift algorithm, $s_{A}$ defined for a given $A$, uses $A$ to solve the problem for the $\ell$ possible partitions and selects the solution that requires minimum number of disks.

The following lemma gives the performance ratio of $s_{A}$ (denoted $r_{s_{A}}$ ) as a function of $\ell$ and the performance ration of $A$ (denoted $\left.r_{A}\right)$.

\section{Lemma 1.8}

$$
r_{s_{A}} \leq r_{A}\left(1+\frac{1}{\ell}\right)
$$

The local algorithm $A$ may itself be derived from an application of the shifting technique. In our example, in order to solve the covering problem on a strip of width $\ell D$, the strip is cut into squares of size $\ell D \times \ell D$, for which an optimal solution can be found by exhaustive search.

We note that the above shifting technique can be used also to derive PTASs for several problems including minimum vertex-cover and maximum independent-set in planar graphs [6]. The idea is that a planar graph can be decomposed into components of bounded outer-planarity. The solution for each component can be found using dynamic programming. The shifting idea is to remove one 'layer' from the graph in each iteration. This removal guarantees that the number of cross-cluster edges is small, so by considering the union of the local cluster solutions one can get a good approximation to the general problem.

\section{Concluding Remarks}

There are many other interesting applications of the techniques described in this chapter. We mention a few of them. Golubchik et al. apply enumeration to a structured instance in solving the problem of data 
placement on disks (see also in [30]). The technique of extending solutions for small subsets is applied by Khuller at al. [34] to the problem of broadcasting in heterogeneous networks. Kenyon et al. [32] used a non-trivial combination of grouping with periodic scheduling to obtain a PTAS for data broadcast.

Some techniques are specialized for certain types of problems. For graph problems, some PTASs exploit the density of the input graph (see, e.g., [5]). There are PTASs which build on the properties of planar graphs (see, e.g., $[22,14])$.

Finally, we have mentioned in Sections 2.3 and 4 some techniques used in randomized approximation schemes. A detailed exposition of randomized approximation schemes for counting problems is given in Chapter 11 in [38] (see also Chapter R-8 in this book). Benczúr and D.R. Karger present in [7] randomized approximation schemes for cuts and flows in capacitated graphs. Efraimidis and Spirakis used in [15] the technique of filtered randomized rounding in developing randomized approximation schemes for scheduling unrelated parallel machines.

\section{References}

[1] F.N. Afrati, E. Bampis, C. Chekuri, D.R. Karger, C. Kenyon, S. Khanna, I. Milis, M. Queyranne, M. Skutella, C. Stein, M. Sviridenko, Approximation Schemes for Minimizing Average Weighted Completion Time with Release Dates. In Proc of FOCS, 32-44, 1999.

[2] S. Arora Polynomial time approximation schemes for Euclidean traveling salesman and other geometric problems. Journal of the ACM, 45(5):753-782, 1998.

[3] S. Arora, Approximation schemes for NP-hard Geometric Optimization Problems: A survey. Math Programming, 2003.

[4] S. Arora and G. Karakostas, Approximation Schemes for Minimum Latency Problems. SIAM J. Comput., 32(5), 1317-1337, 2003.

[5] S. Arora, D, Karger and M. Karpinski. Polynomial Time Approximation Schemes for Dense Instances of NP-Hard Problems. In Proc. of STOC, 1995. 
[6] B.S. Baker. Approximation algorithms for NP-complete problems on planar graphs, J. of ACM, vol. 41(1), pp. 153-180, 1994.

[7] A.A. Benczúr and D.R. Karger. Randomized Approximation Schemes for Cuts and Flows in Capacitated Graphs. Technical Report, MIT, July 2002.

[8] N. Bansal and M. Sviridenko. Two-dimensional bin packing with one dimensional resource augmentation. Submitted for publication.

[9] A. Caprara, H. Kellerer, U. Pferschy, and D. Pisinger. Approximation algorithms for knapsack problems with cardinality constraints. European Journal of Operational Research, 123:333- 345, 2000.

[10] A.K. Chandra, D.S. Hirschberg and C.K. Wong, "Approximate Algorithms for Some Generalized Knapsack Problems". Theoretical Computer Science 3, pp. 293-304, 1976.

[11] C. Chekuri and S. Khanna. On Multidimensional Packing Problems. SIAM J. Comput. 33(4): 837-851, 2004.

[12] C. Chekuri and S. Khanna. A PTAS for the multiple knapsack problem. In Proc. of SODA, 213-222, 2000 .

[13] C. Chekuri and S. Khanna. Approximation Schemes for Preemptive Weighted Flow Time. In Proc. of STOC, 297-305, 2002.

[14] E.D. Demaine and M. Hajiaghayi, "Bidimensionality: New Connections between FPT Algorithms and PTASs," In Proc. of the 16th Annual ACM-SIAM Symposium on Discrete Algorithms (SODA), 590-601, 2005.

[15] P. Efraimidis and P.G. Spirakis. Randomized Approximation Schemes for Scheduling Unrelated Parallel Machines. Electronic Colloquium on Computational Complexity (ECCC), 7(7), 2000.

[16] L. Fleischer, "A Fast Approximation Scheme for Fractional Covering Problems with Variable Upper Bounds". In Proc. of SODA, pp. 994-1003, 2004. 
[17] A. M. Frieze and M.R.B. Clarke, Approximation Algorithms for the m-dimensional 0-1 knapsack problem: worst-case and probabilistic analyses. In European J. of Operational Research, 15(1):100-109, 1984.

[18] W. Fernandez de la Vega, M. Karpinski, C. Kenyon, and Y. Rabani, Approximation Schemes for Clustering Problems. In Proc. STOC'03.

[19] W. Fernandez de la Vega and C. Kenyon. A randomized approximation scheme for metric MAX-CUT. Proc. 39th IEEE Symp. on Foundations of Computer Science, pp 468-471, 1998.

[20] M.R. Garey and R.L. Graham Bounds for Multiprocessor Scheduling with Resource Constraints. SIAM J. Comput. 4(2): 187-200, 1975.

[21] R.L. Graham. Bounds for Certain Multiprocessing Anomalies. Bell Systems Technical Journal, 45:1563$1581,1966$.

[22] M. M. Halldórsson and G. Kortsarz. Tools for Multicoloring with Applications to Planar Graphs and Partial k-Trees. Journal of Algorithms 42(2), 334-366, 2002.

[23] D.S. Hochbaum and D.B. Shmoys. Using dual approximation algorithms for scheduling problems: Practical and theoretical results. Journal of the ACM, 34(1):144-162, 1987.

[24] D.S. Hochbaum. Approximation Algorithms for NP-Hard Problems. PUS Publishing Company, 1995.

[25] D.S. Hochbaum and W. Maass. Approximation schemes for covering and packing problems in image processing and VLSI Journal of the ACM, 32(1):130 - 13, 1985.

[26] P. Indyk. A sublinear time approximation scheme for clustering in metric spaces. In Proc. FOCS '99

[27] K. Jansen, R. Solis-Oba, M. Sviridenko, Makespan Minimization in Job Shops: A Linear Time Approximation Scheme. SIAM J. Discrete Math. 16(2), 288-300, 2003.

[28] K. Jansen, M. Sviridenko, Polynomial Time Approximation Schemes for the Multiprocessor Open and Flow Shop Scheduling Problem. In Proc. of STACS, 455-465, 2000.

[29] D. Karger, C. Stein and J. Wein. Scheduling Algorithms, In CRC Handbook on Algorithms, 1997. 
[30] S. Kashyap and S. Khuller, Algorithms for Non-Uniform Size Data Placement on Parallel Disks. FST \& TCS, 2003

[31] H. Kellerer, U. Pferschy and D. Pisinger. Knapsack Problems. Springer, 2004.

[32] C. Kenyon, N. Schabanel, N.E. Young, Polynomial-Time Approximation Scheme for Data Broadcast. CoRR cs.DS/0205012, 2002.

[33] S. Khanna and R. Motwani, Towards a Syntactic Characterization of PTAS, Proc. of STOC, 329-337, 1996.

[34] S. Khuller, Y. Kim and G. Woeginger, A polynomial time approximation scheme for broadcasting in heterogeneous networks. Proc. of APPROX, 2004.

[35] S. G. Kolliopoulos, "Approximating covering integer programs with multiplicity constraints", Discrete Applied Math., 129:2-3, 461 -473, 2003.

[36] S. G. Kolliopoulos and N. E. Young, "Tight Approximation Results for General Covering Integer Programs". In Proc. of FOCS, 522-528, 2001.

[37] R. Motwani. Lecture notes on approximation algorithms. Technical report, Dept. of Computer Science, Stanford Univ., CA, 1992.

[38] R. Motwani and P. Raghavan. Randomized Algorithms Cambridge Univ. Press, 1995.

[39] C.N. Potts. Analysis of a linear programming heuristic for scheduling unrelated parallel machines. Discrete Applied Mathematics 10, pp.155-164, 1985.

[40] S. Sahni. Approximate Algorithms for the 0/1 knapsack problem, J. of the ACM, 22, 115-124, 1975.

[41] S. Sahni. Algorithms for scheduling independent tasks, J. of the ACM, 23, 555-565, 1976.

[42] P. Schuurman and G.J. Woeginger. Approximation Schemes - A Tutorial. To appear in the book "Lectures on Scheduling", edited by R.H. Möehring, C.N. Potts, A.S. Schulz and G.J. Woeginger, LA Wolsey.

[43] S.V. Sevastianov and G.J. Woeginger. Makespan minimization in open shops: A polynomial time approximation scheme. Mathematical Programming, Vol. 82, 191-198, 1998. 
[44] H. Shachnai, O. Shmueli and R. Sayegh. Approximation Schemes for Deal Splitting and Covering Integer Programs with Multiplicity Constraints, Proc. of WAOA, 111-125, 2004.

[45] H. Shachnai and T. Tamir, "Approximation Schemes for Generalized 2-dimensional Vector Packing with Application to Data Placement". In Proc of APPROX, August 2003.

[46] H. Shachnai and T. Tamir and O. Yehezkely, Approximation Schemes for Packing with Item Fragmentation. 3rd Workshop on Approximation and Online Algorithms (WAOA), October 2005.

[47] V. Vazirani, Approximation algorithms, Springer Verlag, 2001.

[48] G.J. Woeginger, "There is no Asymptotic PTAS for two-dimensional vector packing", Information Processing Letters, 64, pp. 293-297, 1997. 